\title{
Avaliação sensorial de adolescentes com desenvolvimento normal na faixa etária de 11 a 18 anos
}

\author{
Nara Suemi Shishido ${ }^{a}$, Cristina Yoshie Toyida ${ }^{b}$ \\ aTerapeuta ocupacional, Universidade Federal de São Carlos - UFSCar, São Carlos, SP, Brasil

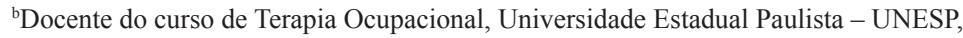 \\ Marília, SP, Brasil, Docente do Programa de Pós-graduação em Educação Especial, \\ Universidade Federal de São Carlos - UFSCar, São Carlos, SP, Brasil
}

\begin{abstract}
Resumo: A sensibilidade tátil contribui para o adequado desenvolvimento neuropsicomotor, possibilita a compreensão do sujeito sobre seu próprio corpo e também de suas ações e explorações sobre os objetos, as pessoas e o meio ambiente. Estudos anteriores avaliaram crianças de 3 a 10 anos de idade e a presente pesquisa aborda o período da adolescência. Os objetivos foram: a) verificar se o rápido crescimento corporal altera o padrão de normalidade da sensibilidade tátil dos adolescentes; b) propiciar aos profissionais da área o uso da caixa sensorial lúdica especialmente criada para atender a faixa etária abordada. Participaram da pesquisa 32 jovens, 16 do sexo masculino e 16 do sexo feminino, com idades entre 11 e 18 anos, estudantes de escola estadual e considerados pertencentes a um grupo padrão de desenvolvimento normal. Por meio dos testes de grafestesia, estereognosia e o teste dos monofilamentos de Semmes-Weinstein foram avaliadas as sensibilidades das faces ventral e dorsal das mãos direita e esquerda. E através de um questionário identificaram-se informações sobre as atividades manuais relevantes neste período. Como resultados, verificaram-se curiosidade e colaboração na pesquisa com o uso das caixas lúdicas; houve alterações da sensibilidade tátil na face dorsal das mãos a partir dos 13 anos de idade e predominantemente no sexo masculino; as principais atividades informadas pelo sexo masculino foram a escrita e esportes, e no sexo feminino foram a escrita e a limpeza da casa. Conclui-se o estudo com sucesso do equipamento elaborado e evidências iniciais sobre possíveis modificações da sensibilidade tátil normal dos jovens.
\end{abstract}

Palavras-chave: Adolescência, Sensibilidade, Terapia Ocupacional.

\section{Sensory assessment of normal adolescents between 11 and 18 years old}

\begin{abstract}
Tactile sensibility allows for adequate neuropsychomotor development. In addition, it enables the understanding of the subject's own body, actions and explorations of objects, people and the environment. Previous studies have evaluated 3 to 10 year-old children. Herein, we evaluate the tactile sensibility in adolescents. The aim of this study was to verify whether the rapid growth of the body changes the normal pattern of tactile sensibility in adolescents. Furthermore, we provide evaluation material that is easy to use, simple, and secure to handle, designed to attend the specific age range studied. Thirty-two young participants between 11 and 18 years old, sixteen males and sixteen females, were evaluated. The adolescents go to public school and present normal development patterns. Graphesthesia, stereognosia, and Semmes-Weinstein monofilament tests were performed to evaluate the sensibility on the ventral and dorsal sides of the right and left hands. A questionnaire was used to identify the relevant activities performed with the hands during the studied period. The adolescents tested were collaborative during the research and presented curiosity regarding the boxes used in the experiment. Results indicated alterations in the tactile sensibility on the dorsal side of the hands from 13-year- old males. The major activities indicated by the adolescents were writing and sports for males and writing and cleaning for females. First evidences of possible changes in the normal tactile sensibility during adolescence were observed in this study
\end{abstract}

Keywords: Adolescence, Sensibility, Occupational Therapy.

Autor para correspondência: Cristina Yoshie Toyida, Universidade Estadual Paulista, Av. Hygino Muzzi, 737, Campus Universitário, CEP 17525-900, Marília, SP, Brasil, e-mail: cristoyoda@gmail.com; narasuemi@gmail.com

Recebido em 7/7/2011; Revisão em 15/5/2012; Aceito em 6/6/2012. 


\section{Introdução}

A adolescência é um período que se estende, aproximadamente, dos 10 aos 19 anos de idade (ORGANIZAÇÃO..., 1995). Neste período de vida observa-se um vasto campo de estudos psicológicos, sociais, conceituais e dos aspectos biológicos - são proeminentes os temas: crescimento e a maturação sexual.

Para Gallahue e Ozmun (2005), o princípio da adolescência é marcado pelo aumento acelerado do peso e da estatura. Sendo que a idade de início, duração e intensidade do surto de crescimento e da maturação sexual irão variar segundo as condiçóes genéticas e influências ambientais. A estimativa do começo deste período pode variar de 11 anos para os meninos e 9 anos para as meninas, e o período de estabilização, respectivamente, 15 anos e 13 anos de idade. O completo crescimento finalizando de maneira lenta até os 18 anos nos meninos e 16 anos nas meninas.

Barbanti e Machado (2007) identificaram no período entre 9 e 16 anos que a idade óssea no sexo feminino é predominantemente maior à idade cronológica. Enquanto no sexo masculino isso só ocorre a partir dos 11 anos. Ou seja, elas se desenvolvem anteriormente ao sexo masculino. Em ambos os sexos a idade óssea e a idade cronológica apresentam tendência de se igualarem próximo à fase adulta.

Desse modo, durante a maturação biológica identificam-se o crescimento e a redefinição do corpo com consequente necessidade da reestruturação da imagem corporal (TURTELLI, 2003).

A imagem corporal, segundo Osório (1992), é uma representação condensada das experiências passadas e presentes, reais ou fantasiadas, conscientes ou inconscientes; ela é a ideia que o indivíduo tem do próprio corpo.

Em nosso organismo, é a sensibilidade somática responsável pela informação sobre o que está acontecendo na superfície e no interior de nosso corpo. E possui as seguintes divisôes: sensibilidade cutânea (tato), que inclui respostas a vários estímulos: pressão, vibração, calor, frio e dor; cinestesia que dá informação sobre a posição e os movimentos do corpo; e sensibilidades viscerais, que informam a respeito das sensaçóes relacionadas aos órgãos internos ou proximidades (CARLSON, 2002).

A imagem corporal é influenciada pela sensibilidade cinestésica, mas também pela sensibilidade tátil através do contato da pele com os objetos, pessoas e o ambiente (TURTELLI, 2003).
A pele oferece a barreira entre o organismo e o ambiente externo. Através dos receptores distribuídos por todas as suas camadas são realizadas as intermediaçôes das sensaçóes de temperatura, dor e tato (CARLSON, 2002).

Após o estímulo ser percebido pelos receptores táteis na pele e para ocorrer a interpretação/ compreensão, este é enviado pelos diferentes órgáos sensoriais até a área somestésica localizada no cérebro. As representaçóes das partes do corpo nesta área ocorrem de acordo com sua importância funcional, por exemplo, as maiores representaçôes nos seres humanos referem-se às mãos (principalmente os dedos), face e boca, onde encontramos também o maior número de receptores táteis (MACHADO, 2003; MAU et al., 1991; BENTZEL, 2007).

Uma pesquisa identificou que as pessoas com dedos menores possuem maior refinamento da sensibilidade tátil. Sendo assim, o sexo feminino apresenta esse sentido mais apurado que o sexo masculino devido ao tamanho dos dedos, frequentemente menor. A explicaçáo decorre da maior aproximaçáo das impressôes digitais, e consequentemente dos diversos receptores entre si que conseguem captar e transmitir ao sistema nervoso central uma informação mais detalhada (PETERS; HACKEMAN; GOLDREICH, 2009).

Uma das mais importantes funçôes da sensibilidade tátil está relacionada com a utilização da mão em atividades de destreza como escrever, pintar, costurar etc. (DELPRAT; MANSAT, 1982).

O sentido tátil também tem relação com aspectos sociais e afetivos. O experimento clássico de Harlow (1958) mostrou que macacos recém-nascidos separados de suas mães biológicas e colocados em gaiolas com duas bonecas, que tinham função de serem mães "adotivas", preferiam a mãe feita com tecido macio, aconchegante e sem alimento para oferecer do que a máe feita de arame mas portadora de uma mamadeira. Harlow (1958) ponderou que alimentação é importante para a sobrevivência, mas o contato tátil é fundamental para que os macacosbebês se desenvolvessem normalmente. As décadas posteriores produziram estudos e investigaçôes que comprovaram os efeitos do toque no desenvolvimento de bebês (HUSS, 1977; POSTHUMA, 1985).

A sensibilidade tátil é um dos componentes corporais que interferem no desempenho funcional do indivíduo nas atividades de vida diárias (autocuidado, trabalho e lazer). Esse sistema influencia a rotina em todos os momentos, principalmente em atividades em que a visão não pode auxiliar eficientemente, por exemplo, saber se o prato retirado do forno de 
microondas está quente, encontrar objetos no bolso, fazer o laço, colocar ou retirar o colar (BENTZEL, 2007).

Como apresentado acima, a sensibilidade tátil interfere de diferentes modos na qualidade de vida humana. O terapeuta ocupacional é um importante profissional nos processos de promoção de saúde, prevenção de doenças ou durante o processo de reabilitação (MELLO; MANCINI, 2007).

A avaliação sensorial pode ser usada para determinar a necessidade de ensinar o paciente a se proteger de lesóes, usar técnicas de compensaçáo como orientação visual para o movimento durante as atividades e se é viável um programa de reeducação sensorial, dessensibilização ou de integração sensorial (FREITAS, 2005)

Um renomado instrumento de avaliação de sensibilidade tátil, térmica e dolorosa é o monofilamento de Semmes-Weinstein. Desde 1970, Bell-Krotoski realizou estudos com comprovaçóes de sua eficiência (FREITAS, 2005).

No Brasil, desde 1983 utiliza-se o kit de monofilamentos de Semmes-Weinstein com 6 monofilamentos, elaborado pela instituição SorriBauru, que atua com pacientes hansenianos, os quais, em geral, apresentam insensibilidade ou deficiências de sensibilidade nas mãos. Os riscos de dor, ferimentos e/ou lesões com uso dos monofilamentos são nulos, o material feito de fios de nylon não perfura a pele (BACCARELLI et al., 1994; FERRIGNO, 2007).

Esse teste tem a vantagem de controlar a força de aplicação sobre a pele, portanto fornecendo sensação protetora, e, além disso, é padronizado permitindo a quantificação, comparação e interpretação de resultados (BACCARELLI et al., 1994; FERRIGNO, 2007).

Durante a avaliação sensorial é imprescindível a obliteração da visão para evitar a compensação dos sentidos (FREITAS, 2005).

Verificou-se em dois estudos sobre a avaliação sensorial em crianças de 3 a 6 anos de idade, e também dos 7 aos 10 anos de idade a importância da confecção de caixas obliteradoras da visão com motivo colorido e lúdico. As caixas constituíram-se num atrativo e reduziram a resistência, medo e ansiedade durante a realização dos testes (JUNQUEIRA; TOYODA, 2002; ARAKAKI; TOYODA, 2006).

Outra questão na literatura sobre a avaliação sensorial refere-se sobre a necessária inclusão de outros testes para melhor avaliação funcional, por exemplo a utilização do teste de estereognosia, que avalia o controle motor e a preensão (MOBERG, 1960 apud TUBIANA; THOMINE; MacKIN, 1996).
Através da revisão da literatura (GALLAHUE; OZMUN, 2005; BARBANTI; MACHADO, 2007; TURTELLI, 2003), verificaram-se diversas pesquisas sobre a adolescência, e o presente estudo pretendeu iniciar a caracterização desse momento da vida em relação à sensibilidade tátil. Além disso, buscou verificar possíveis modificações dessa função, uma vez que estudos anteriores não encontraram evidências de modificações na sensibilidade tátil normal em crianças (JUNQUEIRA; TOYODA, 2002; ARAKAKI; TOYODA, 2006).

Os objetivos do estudo foram: a) Verificar se o rápido crescimento corporal altera o padrão de normalidade da sensibilidade tátil dos adolescentes; b) Propiciar aos profissionais da área o uso da caixa sensorial lúdica especialmente criada para atender a faixa etária abordada.

\section{Metodologia}

Participantes: participaram da pesquisa 32 jovens com idade entre 11 e 18 anos, sendo 4 de cada faixa etária, 2 do sexo feminino e 2 do sexo masculino, estudantes e considerados pertencentes a um grupo padráo de desenvolvimento normal.

A amostra foi definida a partir do tempo hábil e das possibilidades da escola.

Local: escola estadual de ensino fundamental e médio no município de São Carlos, São Paulo, Brasil.

Material e equipamento: o equipamento desenvolvido foi uma caixa de papelão forrada com espuma fina e feltro com duas aberturas onde foram encaixadas as mãos, permitindo que o jovem permanecesse de olhos abertos, mas sem visualizar o teste que se efetuava. Foram construídas duas caixas: uma caixa foi forrada com feltro verde e com detalhes em amarelo e com obliterador da visão como bandeira brasileira, para os jovens do sexo masculino, com o tema Copa do Mundo (Figura 1). Para as jovens do sexo feminino foi construída uma caixa de feltro azul-marinho, com aplicaçóes de flores coloridas (Figura 2).
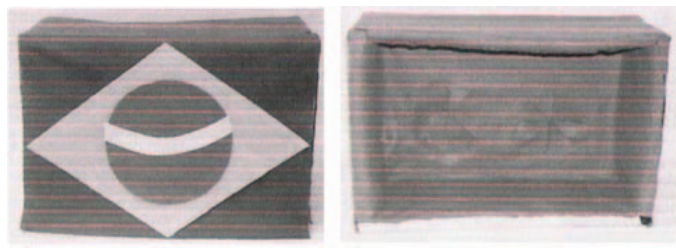

Figura 1. Caixa utilizada com participantes do sexo masculino, respectivamente em vista frontal e posterior. 


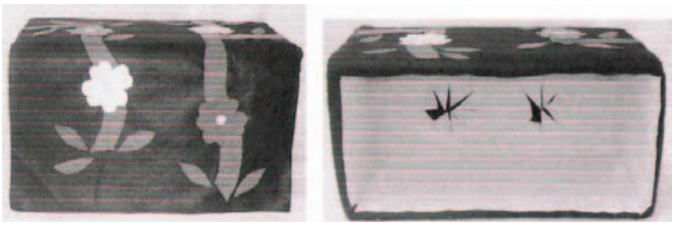

Figura 2. Caixa utilizada com participantes do sexo feminino, respectivamente em vista frontal e posterior.

Foi elaborado um questionário para situar o universo profissional e pessoal dos sujeitos testados e as perguntas incluíam a identificação de três atividades com o uso primordial do sentido tátil de realização mais frequente.

Para avaliação da sensibilidade tátil utilizou-se o teste dos monofilamentos ou estesiômetro (SemmesWeinstein). As anotaçôes foram feitas numa folha de avaliação sensorial que continha um mapeamento detalhado através da distribuição dos pontos nos territórios específicos aos nervos: ulnar, mediano e radial. Os pontos totalizados eram 32, sendo $16 \mathrm{em}$ cada máo, 9 pontos na face palmar e 7 pontos na face dorsal.

O processo de avaliação contou com dois testes não padronizados, mas complementares na avaliação da sensibilidade tátil. Foi utilizado o teste de grafestesia, que analisou o reconhecimento das letras escritas sobre a pele dorsal, testando as seguintes letras: B, J, L, M e N. E também foi empregado o teste de estereognosia, que avaliou o reconhecimento dos objetos através do sentido tátil. Nesse último foi utilizado um recipiente com arroz onde foram colocados os seguintes objetos de metais: clipe, moeda, parafuso, arruela, chave e tampa de caneta.

\section{Procedimento}

Estudo iniciado após aprovação da pesquisa pelo Comitê de Ética em Pesquisa em Seres Humanos da Universidade Federal de São Carlos, CAAE 0257.0.000.135-07, parecer no $174 / 2007$.

Inicialmente foi realizado um teste piloto com 4 jovens. Posteriormente, com as devidas modificaçóes, foi realizado a coleta de dados.

Os testes foram feitos no anfiteatro da escola, sem interferências externas tais como ruídos, entradas e saídas de pessoas que poderiam comprometer a resposta dos jovens, por desviar sua atenção do teste.

Cada aluno entrava individualmente na sala e sentava-se à frente da pesquisadora, que dava as explicaçóes sobre os propósitos do estudo, instrumentos a serem utilizados e assinatura do
Termo de Consentimento Livre e Esclarecido para ele e para o responsável.

Em seguida, iniciou-se o procedimento de coleta de dados com o teste de grafestesia, estereognosia e teste dos monofilamentos de Semmes-Wenstein. Por fim, foi pedido aos estudantes que respondessem ao questionário.

A coleta de dados com cada pessoa durou, em média, 12 minutos. Houve de modo frequente a expressão verbal do medo em relaçáo ao teste dos monofilamentos, o qual era superado quando a pesquisadora demonstrava a sensação que era proporcionada pelo objeto numa área visível como na face palmar de um dos dedos ou no rosto na regiáo zigomática do participante e, desse modo, o jovem verificava o risco nulo de ter ferimentos ou sentir dor. A avaliação foi efetuada num período de 4 dias em alunos dos períodos da manhã, tarde e noite. A escolha dos participantes foi realizada com a ajuda dos professores aos quais foi solicitado que indicassem os alunos com personalidades variadas: introspectivos e extrovertidos, com a finalidade de enriquecer a coleta de dados com uma população diversificada.

\section{Resultados e discussão}

O princípio da pesquisa foi com o teste piloto no qual se identificou a necessidade de modificar o teste de estereognosia, que foi elaborado para a identificação de texturas (veludo, jeans, lixa), objetos (chave, caneta esferográfica, borracha) e formas (quadrados, retângulo). Os jovens apresentaram extrema facilidade em sua realização, não se adequando a intenção das pesquisadoras do fornecimento de um nível de dificuldade, e, portanto, envolvendo desafio e interesse pelos participantes.

Como resultados do estudo, verifica-se o sucesso dos jovens na realização dos testes de grafestesia e de estereognosia, sendo que levaram um tempo maior para a identificação, respectivamente, da letra $\mathrm{J}$ e da arruela. Tais resultados contribuem para a comprovação da amostra deste estudo constituída de jovens com o desenvolvimento normal do controle motor, preensão e sensibilidade tátil (TUBIANA; THOMINE; MacKIN, 1996).

Durante as avaliaçóes, os jovens através de relatos verbais expressaram a curiosidade quanto às caixas temáticas e informaram a identificação com os temas ilustrados. Não houve problemas durante a coleta de dados. Esses resultados corroboram com outros estudos que fizeram uso de material lúdico durante 
a avaliação sensorial (ARAKAKI; TOYODA, 2006; JUNQUEIRA; TOYODA, 2002).

No teste dos monofilamentos de Semmes-Wenstein foi possível identificar a presença de sensibilidade tátil normal (monofilamento verde) e a presença de alteraçóes representadas pela identificação do monofilamento azul que correspondem à sensibilidade diminuída na mão, com dificuldade quanto à discriminação fina (BRASIL, 1997).

$\mathrm{Na}$ face palmar das mãos, direita e esquerda, verificou-se que todos os jovens apresentaram sensibilidade normal (monofilamento verde), exceto uma jovem de 18 anos que identificou 2 pontos estimulados com o monofilamento azul.

$\mathrm{Na}$ região dorsal das máos, Figuras 3 e 4, verificou-se que aproximadamente 10 adolescentes identificaram o toque somente com o monofilamento azul em pelo menos um ponto avaliado do dorso da mão direita e/ou esquerda.

Segundo Carlson (2002) e Tubiana, Thomine e Mackin (1996), a palma possui maior número de receptores sensoriais que o dorso da mão. Desse modo, os resultados desta pesquisa sugerem que a precisão da identificação da sensibilidade tátil pode ter sido afetada pelas características específicas das áreas avaliadas.

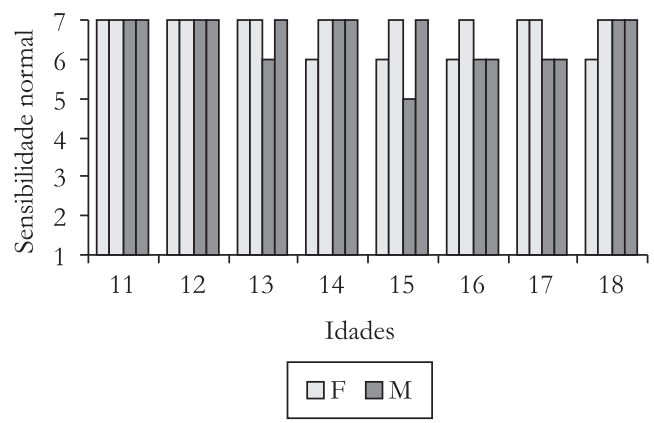

Figura 3. Sensibilidade cutânea da face dorsal direita nos jovens do sexo feminino $(\mathrm{F})$ e do sexo masculino $(\mathrm{M})$.

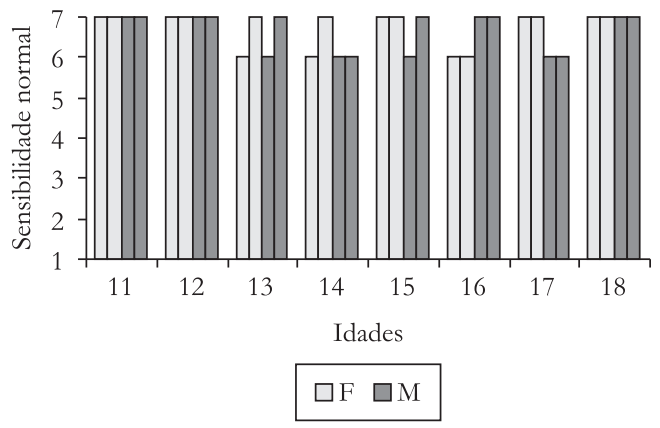

Figura 4. Sensibilidade cutânea da face dorsal esquerda, feminino (F) e masculino (M).
Ainda, nas Figuras 3 e 4, verificou-se que os pontos no dorso da mão identificados com o monofilamento azul totalizaram em 21 pontos de modo uniforme e, portanto, sem ligação com a dominância manual corroborando com o estudo de Andrade et al. (1999) em que não ocorreu alteração significante entre a sensibilidade tátil da mão dominante e da mão não dominante.

Destes 21 pontos, 6 pontos corresponderam ao quarto dedo (anular), sendo 3 pontos localizados na falange média e 3 pontos localizados na falange distal; e 10 pontos corresponderam ao terceiro dedo (médio), localizados na falange distal.

$\mathrm{Na}$ literatura, o uso do dedo anular relaciona-se com atividades que demandam a preensão palmar de força. Esta envolve a regiáo palmar de todos os dedos, sendo que a face palmar do polegar inervada pelo nervo mediano pode, dependendo do diâmetro do objeto, entrar em contato com a pele dorsal das falanges médias ou falanges distais dos dedos indicador e/ou médio, também inervada pelo nervo mediano. Alguns exemplos são: segurar objetos pesados e volumosos, por exemplo, vassoura e martelo (KAPANDJI, 2000; TUBIANA; THOMINE; MacKIN, 1996).

O dedo médio participa nas pinças de força (por exemplo: abrir a tampa da pasta de dente, segurar uma bola de pingue-pongue, desenroscar a tampa de um pote) e na pinça de precisão (por exemplo: segurar um fio, escrever e pintar com pincel) (KAPANDJI, 2000; TUBIANA; THOMINE; MacKIN, 1996).

A Figura 5 representa as mudanças da sensibilidade cutânea em relação aos gêneros com o decorrer dos anos.

Nessa figura verificou-se que houve total identificação do monofilamento verde até os 12 anos de idade. Contudo, com o aumento da idade dos participantes, a partir dos 13 anos e principalmente no sexo masculino, ocorreu, em alguns pontos do

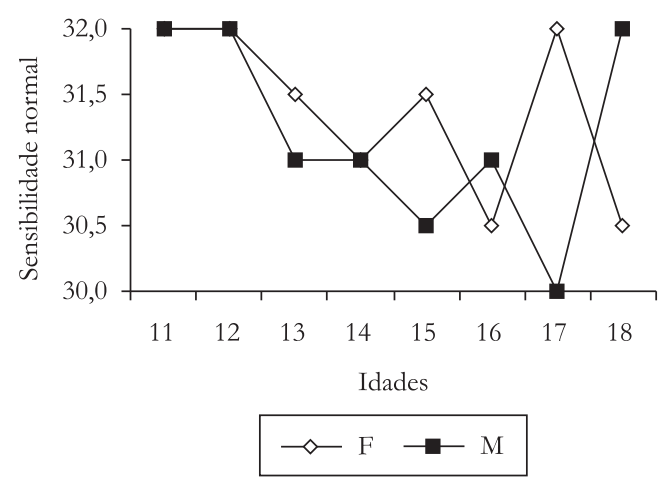

Figura 5. Alterações da sensibilidade cutânea em relação aos gêneros com o decorrer dos anos. 
Tabela 1. Principais atividades realizadas pelos adolescentes.

\begin{tabular}{lcccc}
\hline \multicolumn{2}{c}{ Meninas } & & \multicolumn{2}{c}{ Meninos } \\
\hline Atividades & Número de sujeitos & & Atividades & Número de sujeitos \\
\hline Escrita & 16 & & Escrita & 13 \\
Limpeza doméstica & 11 & & Esportes & 13 \\
Outros & 9 & Outros & 8 \\
\hline
\end{tabular}

dorso da mão, o monofilamento azul, indicando possivelmente alguma modificação sensorial.

E possível com a literatura hipotetizar, e relacionar o resultado acima devido ao período do surto de crescimento e possíveis alteraçóes da percepção dos membros e da imagem corporal. Nessa fase ocorre a reestruturação e unificação das sensaçóes do corpo em desenvolvimento, enquanto isso os jovens são caracterizados por dificuldade ou falta de fluidez e destreza na habilidade de sentir e de se movimentar (TURTELLI, 2003; GALLAHUE; OZMUN, 2005).

Kestenberg, em 1975, apud Turtelli (2003), pesquisadora dos padróes motores, identificou nas fases pré-púbere, crescimento da puberdade e diferenciação da puberdade que os movimentos corporais apresentam limitaçóes na dissociação entre cabeça, tronco e membros, e desse modo demonstram desorganização na sua atitude e ritmicidade. Já durante o período de consolidação pré-adulta é caracterizado pela solidificação das fronteiras do corpo e consequente melhora do posicionamento e harmonia do corpo no espaço (TURTELLI, 2003).

Através da análise dos questionários respondidos pelos adolescentes verificaram-se as tarefas manuais elegidas pelos jovens como predominantes nessa fase. Constataram-se nos questionários 96 indicações de atividades em que o sentido tátil fosse predominante (considerando-se que 32 pessoas participaram da pesquisa e cada uma deveria indicar 3 atividades), ou seja, 48 no sexo feminino e 48 no sexo masculino. $\mathrm{Na}$ Tabela 1 observam-se as principais atividades da rotina dos adolescentes.

Em ambos os sexos a escrita foi apontada como a principal atividade que se relaciona com o sentido tátil. As diferenças foram verificadas nas demais atividades; para o sexo feminino a atividade de limpeza doméstica e no sexo masculino foi informada a prática de esportes de modo relevante e igualitária à atividade de escrita.

A diferença de gênero e realização de atividades foi discutida no estudo de Lima et al. (2006). Independente de classe social, os meninos se relacionaram predominantemente com as atividades exteriores (tarefas realizadas fora do domínio da casa, como comprar produtos, limpar quintal, jogar o lixo fora). Enquanto as meninas informaram a atividade de alimentação (tarefas de preparo ou ajuda na preparação de refeiçôes da casa).

Resumindo, hipotetiza-se que os resultados desta pesquisa, embasados na literatura, possivelmente foram influenciados pelos fatores: realização de atividades segundo o gênero que podem interferir em diferentes ganhos de habilidades no desenvolvimento da sensibilidade tátil (DELPRAT; MANSAT, 1982; PETERS; HACKERMAN; GOLDREICH, 2009).

E ainda, apesar dessa alteração ter ocorrido na face dorsal que não está relacionada diretamente com o toque em objetos, estudos verificaram que em algumas preensóes temos o contato da face dorsal dos dedos com a face palmar das máos (TUBIANA; THOMINE; MacKIN, 1996; KAPANDJI, 2000). Portanto, hipotetiza-se que poderia ocorrer o atrito das estruturas e formaçáo de calosidades dependendo do padrão da escrita ou do esporte realizado que não foram detalhados neste estudo; associada à fase de surto de crescimento que pode levar a alterações da percepção corporal (TURTELLI, 2003; GALLAHUE; OZMUN, 2005; BARBANTI; MACHADO, 2007) e ainda integrada pela questáo fisiológica e funcional de redução de receptores sensoriais na face dorsal das mãos (CARLSON, 2002; TUBIANA; THOMINE; MacKIN, 1996) resultaram possivelmente nas alterações de sensibilidade no período da adolescência, ou seja, a sensibilidade diminuída na região dorsal das mãos, iniciando aos 13 anos e predominantemente no sexo masculino. Entretanto, devido ao número reduzido de participantes novos estudos fazem-se necessários.

\section{Conclusão}

Nesta pesquisa participaram pessoas sem queixas e sem o diagnóstico de alteraçôes de sensibilidade tátil, ou seja, com o desenvolvimento da sensibilidade normal.

As caixas utilizadas como equipamentos para obliterar a visão cumpriram com o propósito de não oferecer intimidação e facilitar o processo de avaliação.

Os resultados demonstraram a sensibilidade tátil normal na face palmar e a partir dos 13 anos 
verificou-se a diminuição da sensibilidade tátil na face dorsal das mãos, principalmente no sexo masculino.

Através do questionário constatou-se que a escrita foi a atividade mais referida pelos jovens no uso da sensibilidade tátil, seguida pelas tarefas domésticas no sexo feminino e esportes no sexo masculino.

Esta pesquisa apresentou a colaboração dos jovens com o equipamento utilizado e possíveis evidências iniciais de modificaçóes da sensibilidade tátil normal no período da adolescência.

\section{Referências}

ANDRADE, L. B. et al. Diferença de sensibilidade entre mãos dominante e não-dominante testada com os monofilamentos de Semmes-Weinstein. Reabilitar, São Paulo, v. 2, n. 5, p. 37-41, 1999.

ARAKAKI, V. C.; TOYODA, C. Y. Avaliação sensorial em crianças de sete a dez anos de idade e o uso do lúdico como recurso em Terapia Ocupacional. São Carlos: UFSCar, 2006. Relatório Final do PIBIC CNPq/UFSCar.

BACCARELLI, R. et al. Perguntas e respostas sobre o teste de sensibilidade com os monofilamentos de nylon de Semmes-Weintein (S-W). Bauru: [s.n.], 1994.

BRASIL. Ministério da Saúde. Fundação Nacional de Saúde. Manual de Prevenção de Incapacidades. Brasília: Fundação Nacional de Saúde, 1997.

BARBANTI, V. J.; MACHADO, D. R. L. Maturação esquelética e crescimento em crianças e adolescentes. Revista Brasileira de Cineantropometria e Desempenho Humano, Florianópolis, v. 9, n. 1, p. 12-20, 2007.

BENTZEL, K. Avaliando habilidades e capacidades: a sensibilidade. In: TROMBLY, C. A.; RADOMSKI, M. V. Terapia Ocupacional para disfunçôes fisicicas. 5. ed. Tradução de Beatriz Bittencourt G. Schlecht e colaboradores. Santos: Santos Editora, 2007. cap. 6, p. 159-175.

CARLSON, N. R. Fisiologia do comportamento. 7. ed. São Paulo: Editora Manole, 2002. p. 221-222.

DELPRAT, J.; MANSAT, M. La réducation de la sensibilité - un problème de tous les tours bien souvent negligé. Journal d'Ergothérapie, [s.n.], v. 4, p. 98-106, 1982.

FERRIGNO, I. S. V. Terapia da mão: Fundamentos para a prática clínica. Santos: Livr.Editora, 2007.

FREITAS, P. P. Reabilitação da mão. Sáo Paulo: Atheneu, 2005.

GALLAHUE, D. L.; OZMUN, J. C. Crescimento do Adolescente, Puberdade e Maturidade Reprodutiva. In: GALlAHUE, D. L.; OZMUN, J. C. Compreendendo o desenvolvimento motor: bebês, crianças, adolescentes e adultos. São Paulo: Phorte, 2005. p. 348-366.

HARLOW, H. F. The nature of love. American Psychologist, Washington, v. 13, p. 673-685, 1958.
HUSS, A. J. Touch with care or a caring touch? American Journal of Occupational Therapy, New York, v. 31, p. 11-18, 1977.

JUNQUEIR A, J. T. S.; TOYODA, C. Y. Avaliação sensorial em crianças de 3 a 6 anos de idade e o uso do lúdico como recurso em Terapia Ocupacional. São Carlos: UFSCar, 2002. Relatório Final do PUIC/UFSCar.

KAPANDJI, A. I. A Mão. In: KAPANDJI, A. I. Fisiologia articular: esquemas comentados de mecânica humana. 5. ed. São Paulo: Panamericana, 2000. cap. 5, p. 174-287.

LIMA M. B. S. et al. Tarefas domésticas e socialização na perspectiva de adolescentes de classe economicamente distintas. In: SEMINÁRIO DE INICIAÇÃO CIENTÍFICA DA UFPA, 16., 2006, Belém. Revista Científica da UFPA, Belém, v. 5, n. 1, p. 1-41, 2006. Disponível em: <http://www.cultura.ufpa.br/rcientifica/ relat_finais/arquivos_pdf/565.pdf >. Acesso em: 27 jul. 2007.

MACHADO, A. Estrutura e funçôes do córtex cerebral. In: MACHADO, A. Neuroanatomia Funcional. 2. ed. São Paulo: Atheneu, 2003. p. 257-273.

MAU, L. L. et al. Evaluación y reeducación de la sensibilidad de la mano en enfermos de lepra. In: TERAPIA Ocupacional: el camino del hacer. Buenos Aires: C.O.L.T.O, 1991. p. 74-108.

MELLO, M. A. F.; MANCINI, M. C. Métodos e Técnicas de Avaliação nas Áreas de Desempenho Ocupacional. In: CAVALCANTI, A.; GALVĀO, C. Terapia Ocupacional: Fundamentaçáo e prática. Rio de Janeiro: GuanabaraKoogan, 2007. p. 49-55.

ORGANIZAÇÃO PANAMERICANA DE LA SALUD - OPS. La Salud Del Adolescente y Del joven. Washington: OPS/OMS, 1995. Publicación científica:552. OSÓRIO, L. C. Adolescente hoje. 2. ed. Porto Alegre: Artes Médicas, 1992.

PETERS, R.; HACKERMANE, E.; GOLDREICH, D. 2009. Diminutive digits discern delicate details: fingertip size and the sex difference in tactile spatial acuity. Journal of Neuroscience, Baltimore, v. 29, n. 50, p. 15756-15761. http://dx.doi.org/10.1523/ JNEUROSCI.3684-09.2009

POSTHUMA, B. W. Learning to touch. Canadian Journal of Occupational Therapy, Toronto, v. 52, n. 14, p. 189-193, 1985.

TUBIANA, R.; THOMINE, J. M.; MacKIN, E. Anatomia Funcional. In: MacKIN, E.; THOMINE, J. M.; TUBIANA, R. Diagnóstico Clínico da mão e do punho. 2. ed. Rio de Janeiro: Interlivros, 1996. cap. 1, p. 1-177.

TURTELLI, L. S. Relaçōes entre imagem corporal e qualidade de movimento: uma reflexão a partir de uma pesquisa bibliográfica. 2003. 332 f. Dissertação (Mestrado)Faculdade de Educação Física. Universidade Estadual de Campinas, Campinas, 2003.

\section{Contribuição dos Autores}

Nara Suemi Shishido: elaboração do estudo, redação do texto, revisão e organização das fontes. Cristina Yoshie Toyoda: revisão do texto. 